\title{
OBSERVATIONAL EVIDENCE OF TORUS INSTABILITY AS TRIGGER MECHANISM FOR CORONAL MASS EJECTIONS: THE 2011 AUGUST 4 FILAMENT ERUPTION
}

\author{
F. P. Zuccarello ${ }^{1,2}$, D. B. Seaton ${ }^{2}$, M. Mierla $^{2,3}$, S. Poedts ${ }^{1}$, L. A. Rachmeler ${ }^{2}$, \\ P. ROMANO ${ }^{4}$, AND F. ZUCCARELlO ${ }^{5}$ \\ ${ }^{1}$ Centre for mathematical Plasma-Astrophysics, KU Leuven, Celestijnenlaan 200B, 3001 Leuven, \\ Belgium; Francesco.Zuccarello@wis.kuleuven.be, Stefaan.Poedts@wis.kuleuven.be \\ ${ }^{2}$ Royal Observatory of Belgium, Ringlaan 3,1180 Brussels, Belgium; dseaton@oma.be, marilena@oma.be, rachmeler@oma.be \\ ${ }^{3}$ Institute of Geodynamics of the Romanian Academy Jean-Louis Calderon 19-21, RO-020032 Bucharest, Romania \\ ${ }^{4}$ INAF-Osservatorio Astrofisico di Catania, via S. Sofia 78, I-95123 Catania, Italy; Paolo.Romano@ oact.inaf.it \\ ${ }^{5}$ Dipartimento di Fisica e Astronomia—Universitá di Catania Via S.Sofia 78, I-95123 Catania, Italy; fzu@ oact.inaf.it \\ Received 2013 November 12; accepted 2014 January 14; published 2014 ???
}

\begin{abstract}
Solar filaments are magnetic structures often observed in the solar atmosphere and consist of plasma that is cooler and denser than their surroundings. They are visible for days—even weeks-which suggests that they are often in equilibrium with their environment before disappearing or erupting. Several eruption models have been proposed that aim to reveal what mechanism causes (or triggers) these solar eruptions. Validating these models through observations represents a fundamental step in our understanding of solar eruptions. We present an analysis of the observation of a filament eruption that agrees with the torus instability model. This model predicts that a magnetic flux rope embedded in an ambient field undergoes an eruption when the axis of the flux rope reaches a critical height that depends on the topology of the ambient field. We use the two vantage points of the Solar Dynamics Observatory $(S D O)$ and the Solar TErrestrial RElations Observatory to reconstruct the three-dimensional shape of the filament, to follow its morphological evolution, and to determine its height just before eruption. The magnetograms acquired by $S D O /$ Helioseismic and Magnetic Imager are used to infer the topology of the ambient field and to derive the critical height for the onset of the torus instability. Our analysis shows that the torus instability is the trigger of the eruption. We also find that some pre-eruptive processes, such as magnetic reconnection during the observed flares and flux cancellation at the neutral line, facilitated the eruption by bringing the filament to a region where the magnetic field was more vulnerable to the torus instability.
\end{abstract}

Key words: methods: observational - Sun: corona - Sun: coronal mass ejections (CMEs) Sun: filaments, prominences

Online-only material: animation, color figures

\section{INTRODUCTION}

Solar filaments are dark, filamentary structures constituted of plasma that is cooler and denser than their surroundings. Although the precise topology of the magnetic configuration suitable for supporting solar filaments is still under debate, magnetic flux ropes, with their shear, twist, and presence of dips, are among the most promising candidates (Mackay \& van Ballegooijen 2009).

Solar filaments are generally visible for days-even weeks-before disappearing or erupting, which suggests that most of the time they are in equilibrium with their environment. The eruption of a filament is the manifestation of the sudden loss of this equilibrium. Understanding what causes (or triggers) the disruption of the equilibrium and drives filament eruptions and their associated coronal mass ejections is still an area of active research.

Several coronal mass ejection initiation models have been proposed (see Forbes 2000, 2010; Klimchuk 2001; Roussev \& Sokolov 2006; Forbes et al. 2006; Chen 2011, for a review). Among the different coronal mass ejection initiation scenarios, one that relies on an ideal-magnetohydrodynamic instability to trigger the eruption is the torus instability model (Bateman 1978; Kliem \& Török 2006). In this model, a current ring of major radius $R$ is embedded in an external magnetic field. Current rings are subjected to a radially outward-directed hoop force that decreases when the ring expands. If the inward- directed Lorentz force caused by the external field decreases faster with $R$ than the hoop force, the system is unstable to perturbations.

Assuming an external field $B_{\text {ex }} \propto R^{-n}$, where $n=$ $-R d\left(\ln B_{\text {ex }}\right) / d R$, Bateman (1978) showed such an instability will occur when $n>n_{\text {crit }}=1.5$. In other words, if the current ring has a major radius $R$ such that the decay index of the external field, $n$, is significantly smaller than $n_{\text {crit }}$, the system is in a stable equilibrium where the inward magnetic tension of the external field balances the outward magnetic pressure of the current channel. However, when $n$ approaches $n_{\text {crit }}$, this equilibrium becomes unstable and any displacement of the current channel caused by some perturbation will initiate an outward motion of the current ring.

Török \& Kliem (2007) performed numerical magnetohydrodynamic simulations of Titov \& Démoulin-type flux ropes (T\&D; Titov \& Démoulin 1999) embedded in an external field. Török \& Kliem considered semicircular flux ropes with a small aspect ratio and confirmed that the torus instability occurs when the flux rope axis reaches a height where the decay index of the external field is larger than $n_{\text {crit }}$. They also found that the steeper the increase of the decay index is-i.e., the faster the external field decreases with height - the larger the initial acceleration of the flux rope is.

In addition, Démoulin \& Aulanier (2010) have shown that the torus instability model is the three-dimensional counterpart of the catastrophic loss of equilibrium model first introduced by 
van Tend \& Kuperus (1978) and later generalized by Forbes \& Isenberg (1991) and Isenberg et al. (1993) to include the effect of finite coronal currents and photospheric line-tying. Starting from the model of Isenberg et al. (1993), Forbes \& Priest (1995) studied a two-dimensional coronal flux rope embedded in the field generated by two photospheric magnetic sources, which undergo convergence motion toward the polarity inversion line that separates them. These authors found that as the distance between the two magnetic sources decreases, the magnetic energy stored in the flux rope increases until the system reaches a critical point at which it experiences a loss of equilibrium and erupts. When the loss of equilibrium occurs, a current sheet is formed below the flux rope that eventually finds a new equilibrium. If magnetic reconnection is allowed, the flux rope undergoes a full eruption (Lin \& Forbes 2000).

Flux cancellation at the polarity inversion line caused by convergence motions has also been studied using three-dimensional magnetohydrodynamic simulations (Amari et al. 2003, 2011; Zuccarello et al. 2012a). In these models, magnetic reconnection occurs between highly sheared field lines in the proximity of the polarity inversion line as a consequence of the convergence flows. This reconnection allows the system to change from an arcade-like to a flux rope-like configuration that eventually experiences a full eruption. As the eruption progresses, the magnetic reconnection below the flux rope, generally referred to as tether-cutting reconnection, has two effects: it reduces the magnetic tension of the overlying field and it also increase the magnitude of the poloidal field of the flux rope.

Aulanier et al. (2010) performed a similar simulation, but the effect of the flux dispersion was obtained by means of increased photospheric diffusion. The authors of that study found that tether-cutting reconnection is important for forming the flux rope and facilitates its slow rise, but the actual trigger of the eruption is the torus instability. If the tether-cutting reconnection is stopped before the critical height for the onset of the torus instability is reached, no eruption is observed. Once the flux rope begins to accelerate, magnetic reconnection begins to occur below it, eventually transferring the overlying field into the flux rope field and resulting in a positive feedback that leads to a full eruption.

However, tether-cutting reconnection is not the only mechanism that can facilitate solar eruptions. For example, Seaton et al. (2011) observed a flow of cold plasma in the low corona just before the eruption that occurred on 2010 April 3. Using stereoscopic triangulation on observations from SWAP onboard PROBA2 and SECCHI onboard the Solar Terrestrial Relations Observatory (STEREO), Seaton et al. reconstructed the threedimensional evolution of the event and concluded that the initial mass off-loading process facilitated the rise of the flux rope, but the eruption itself was likely triggered by the catastrophic loss of equilibrium of the flux rope. Zuccarello et al. (2012b) extended this analysis by investigating the evolution of the magnetic field. Zuccarello et al. concluded that the eruption was compatible with the torus instability scenario-i.e., they found that the estimated location of the flux rope axis was in a region where the decay index was close to $n_{\text {crit }}$. As a result, the increase in the height of the flux rope after the mass off-loading may have been critical to facilitating the flux rope's crossing of the instability threshold, eventually resulting in the full eruption of the filament.

In this paper, we present the analysis of a filament eruption that occurred on 2011 August 4 in National Oceanic and Atmospheric Administration active region 11261 whose inter- pretation supports the eruption scenario described by Aulanier et al. (2010) and Zuccarello et al. (2012a).

Active region 11261 was composed of a group of three sunspots characterized by strong coherent negative polarities. The sunspots were trailed to the east by an extended facula associated with a positive magnetic flux distribution. Also, to the west of the northernmost sunspot there were several pores associated with positive magnetic field. The active region contained several filaments, but in this study we focus on the filament located along the polarity inversion line between the northernmost negative sunspot and the facula.

On 2011 August 3 at 13:17 UT (hereafter referred to as August 3 at 13:17), an M6.0 flare occurred very close to the western footpoint of the filament without destabilizing it. Our analysis shows that at the moment of the M6.0 flare, the filament was torus stable. After the flare, we observed a change in the morphology of the filament as its western footpoint moved southward.

On August 4 at 03:48 the filament erupted completely. In the $14 \mathrm{hr}$ between the M6.0 flare and the filament eruption, we observed flux cancellation at the polarity inversion line driven by converging flows. During this flux cancellation process we also observed several brightenings in the hotter Solar Dynamics Observatory (SDO)/Atmospheric Imaging Assembly (AIA) channels, suggesting the occurrence of tether-cutting reconnection driven by flux cancellation just like in the simulations of Aulanier et al. (2010) and Zuccarello et al. (2012a).

Combining three-dimensional reconstructions of the filament and potential magnetic field extrapolations we show in this paper that the trigger of the eruption was the torus instability. Our analysis also shows that the change in the morphology of the filament and the observed flux cancellation were fundamental to facilitating the eruption. As a result of this morphological change, the filament extended further south, into a region where the filament was more vulnerable to the torus instability. In this new configuration, the flux cancellation, as suggested by the simulation of Aulanier et al. (2010), removed part of the linetying allowing the rise of the filament up to the height where the decay index is larger then $n_{\text {crit }}$, eventually resulting in an eruption.

\section{OBSERVATIONS}

To investigate the coronal evolution of active region 11261 , we used images acquired by the AIA Lemen et al. (2012) on board the SDO Pesnell et al. (2012) with a pixel resolution of about 0 .' 6 and a cadence of $12 \mathrm{~s}$. For this study, we used images acquired between August 3 at 01:42 and August 4 at 03:48 in the $304 \AA, 193 \AA$, and $131 \AA$ passbands.

The face-on view of the Sun provided by SDO/AIA is complemented by views from the side-with increasing angular separation over the past few years-from the Extreme Ultraviolet Imagers (EUVI; Wuelser et al. 2004) on board the twin STEREO Kaiser et al. (2008) spacecraft. On the day of the eruption, the separation angles between Earth and STEREO-A and $-B$ were $100^{\circ}$ and $93^{\circ}$, respectively. Given that the erupting filament was located at a longitude of about $37^{\circ} \mathrm{W}$, the eruption was entirely obscured by the limb of the Sun from the vantage point of STEREO-B. Therefore, we used only the observations provided by $S T E R E O-A$. In particular, we used images acquired by EUVI-A between August 3 at 06:35 and August 4 at 03:46 in the $195 \AA$ and $304 \AA$ passbands. EUVI-A images have a pixel 
Table 1

List of the GOES Flares

\begin{tabular}{|c|c|c|c|}
\hline Day & $\begin{array}{l}\text { Time } \\
\text { (UT) }\end{array}$ & GOES Flare (Beg, Max, End) & Description \\
\hline 2011 Aug 3 & 03:40 & M1.1 (03:08, 03:37, 03:51) & Figure 1(A): the two filaments are visible. \\
\hline 2011 Aug 3 & $04: 29$ & M1.7 (04:29, 04:32, 04:35) & Flare occurred in the nearby active region. \\
\hline 2011 Aug 3 & 06:42 & C1.1 (06:42, 06:46, 06:49) & Figure 1(B): the small filament is activated. \\
\hline 2011 Aug 3 & $07: 38$ & C8.7 (07:38, 07:58, 08:06) & Occurred in the southern part of the active region. \\
\hline 2011 Aug 3 & 10:01 & C1.0 (10:01, 10:04, 10:06) & Occurred in the southern part of the active region. \\
\hline 2011 Aug 3 & 15:08 & $\cdots$ & Figure 1(D): morphological change of the bigger filament. \\
\hline 2011 Aug 3 & $18: 52$ & C2.3 (18:52, 19:00, 19:06) & Flare occurred in the nearby active region. \\
\hline 2011 Aug 3 & 20:02 & C8.5 (19:23, 19:30, 19:42) & Figure 1(E): flare occurred close to the filament footpoint. \\
\hline 2011 Aug 4 & $00: 47$ & C1.7 (00:47, 01:09, 01:23) & Occurred in the southern part of the active region. \\
\hline 2011 Aug 4 & $02: 14$ & C3.2 (02:14, 02:23, 02:28) & Occurred in the southern part of the active region. \\
\hline 2011 Aug 4 & 03:48 & M9.3 (03:41 03:57 04:04) & Figure 1(F): the bigger filament erupts. \\
\hline
\end{tabular}

Note. The key events in the evolution of the active region are in boldface.

resolution of about $1^{\prime \prime} .6$, and, on the date of the eruption, were acquired with a time cadence of 5 minutes in the $195 \AA$ passband and 10 minutes in the $304 \AA$ passband.

We also used full-disk $\mathrm{H} \alpha$ images with a spatial resolution of $1^{\prime \prime}$ acquired by the Big Bear Solar Observatory on August 2 and August 3 to infer the morphology of the filament.

To analyze the magnetic configuration of the active region, we used full-disk line-of-sight magnetograms from the Heliospheric and Magnetic Imager (HMI; Schou et al. 2012) at $6767.8 \AA$ with a pixel resolution of $00^{\prime \prime} 5$ and a temporal resolution of 12 minutes. We used magnetograms acquired between $\mathrm{Au}$ gust 3 at 00:00 and August 4 at 03:48. All of the magnetogram data were corrected for the angle between the magnetic field direction and the observer's line of sight and were co-aligned by applying the standard differential rotation rate reported by Howard et al. (1990).

\section{ANALYSIS}

Active region 11261 first appeared on the east limb of the Sun on July 27 and, during its passage across the solar disk, was the source of several C- and M-class flares. Table 1 includes a list of the flares that occurred during the period of observation, from August 3 at 02:00 until August 4 at 04:00. The key events in the evolution of the active region are indicated in boldface. Figure 1 shows the Geostationary Operational Environmetal Satellite (GOES) X-ray flux (Stern et al. 2004) for the same time interval together with representative AIA $193 \AA$ images. The acquisition times of the AIA images shown in the upper rows are indicated by vertical lines in the GOES flux plot, corresponding to the boldface rows in Table 1.

Figure 1(A) shows the configuration of the active region on August 3 at 03:40. At this time, two filaments (indicated by arrows) are visible: a big cusp-shaped filament and smaller one to the southwest of it.

On August 3 at 06:42, the smaller filament was activated (Figure 1(B)) and part of it eventually erupted, on August 3 at 13:17, resulting in a halo coronal mass ejection and in an M6.0 flare (Figure 1(C)) that is characterized by a long decay phase and by the presence of intense postflare loops (Figure 1(D)).

As we will show in the following subsections, this M6.0 flare represents a milestone in the evolution of the active region. Following the M6.0 flare, there is a clear change in the bigger filament's morphology. The now-larger filament extended farther south into the region where the smaller filament was previously observed (Figures 1(D)-(E)). The filament remained in this new configuration until August 4 at 03:48, when it was activated and underwent a complete eruption. Figure 1(F) shows the early phase of the eruption, highlighting the twisted structure of the flux rope that probably supported the filament material.

\subsection{Filament Morphology}

The most significant change in the morphology of the filament occurred during the M6.0 flare, so to further investigate the evolution of the filament, we considered images taken $12 \mathrm{hr}$ before and after the flare. Figure 2 shows the $\mathrm{H} \alpha$ and the AIA $193 \AA$ images of the active region together with the relevant HMI magnetograms before (top panels) and after (bottom panels) the M6.0 flare. The images have been co-aligned using the mapping software available in SolarSoft.

The $\mathrm{H} \alpha$ image acquired on August 2 at 23:01 shows the presence of a cusp-shaped filament that extends from the eastern facula up to the north of the sunspot. To the west of the sunspot, a smaller filament is visible as a dark, threaded structure that crosses the facula. Our analysis of contemporaneous AIA images confirms the presence of two separate filaments. The western footpoint of the bigger filament seems to cross the eastern footpoint of the smaller filament.

Figure 2(C) shows the contours of the filaments derived from the $193 \AA$ image (red lines) superimposed on the HMI magnetogram. The eastern footpoint of the bigger filament is anchored in the dispersed positive flux distribution of the eastern facula, while the western footpoint is anchored in the negative polarity of the sunspot. Meanwhile, the eastern footpoint of the smaller filament seems to be anchored in the positive flux distribution of the northern sunspot, while its western footpoint appears to be anchored in a negative flux intrusion at the south of it.

The crossing of the footpoints of the two filaments, together with their magnetic configuration, may suggest that the two filaments are actually part of the same complex flux rope in which a significant amount of flux is anchored in the photosphere, close to the location where the two filaments cross each other.

The morphology of the active region after the M6.0 flare is shown in the bottom panels of Figure 2. The $\mathrm{H} \alpha$ image is saturated by the emission of the facula, so it is not possible to clearly determine the morphology of the filament across the facular region. However, the $193 \AA$ image shows the presence of a single arch-shaped filament that extends from the positive polarity 

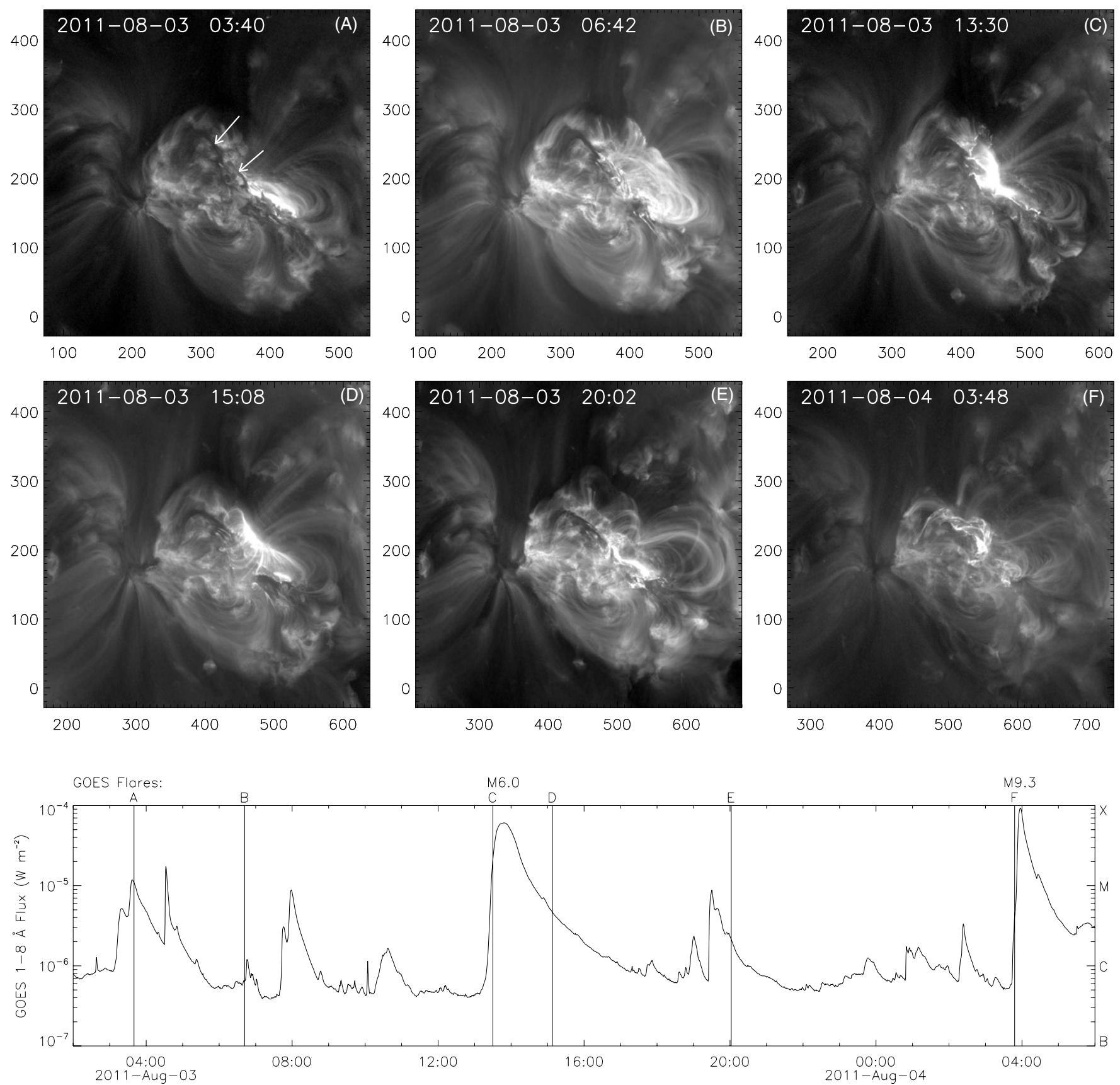

Figure 1. Sequence of co-aligned SDO/AIA $193 \AA$ images acquired between 2011 August 3 03:40 UT and 2011 August 4 03:48 UT (top rows) and integrated X-ray (1-8 A) flux as measured by the X-ray Flux Monitor onboard the GOES 15 spacecraft between 2011 August 3 00:00 UT and 2011 August 4 06:00 UT (bottom panel). The vertical lines in the GOES flux indicate the times at which the AIA $193 \AA$ Amages were obtained. The white arrows in the top-left image indicate the locations of the two filaments. North is at the top of the image; west is to the right.

(An animation of this figure is available in the online journal.)

of the eastern facula to the negative polarity at the south end of the northernmost sunspot. In this new configuration, the axis of the filament extends south along the polarity inversion line between the negative flux of the sunspot and the positive flux to the west of it, where the eastern leg of the smaller filament was anchored before the M6.0 flare.

\subsection{Magnetic Flux}

During the $28 \mathrm{hr}$ that preceded the filament eruption, the photospheric magnetic field evolved considerably, especially close to the location where the footpoints of the two filaments cross each other (see Figure 2(C)-(F)). To analyze the evolution of this magnetic field, in the sequence of co-aligned magnetograms we identified the three subregions that are shown in Figure 3.

The region enclosing all the sunspots of the active region is labeled as Region C, while Region A is a subfield of Region $\mathrm{C}$ centered around the northern sunspot. Finally, the location where the footpoints of the filaments cross each other is labeled as Region A2.

In Region $\mathrm{C}$ we calculated the positive, negative, and total unsigned fluxes by integrating over the full region. However, this approach could not be used for Region A and Region A2. The magnetic field in these regions is quite dynamic, so during the $28 \mathrm{hr}$ during which we tracked the field evolution some of 

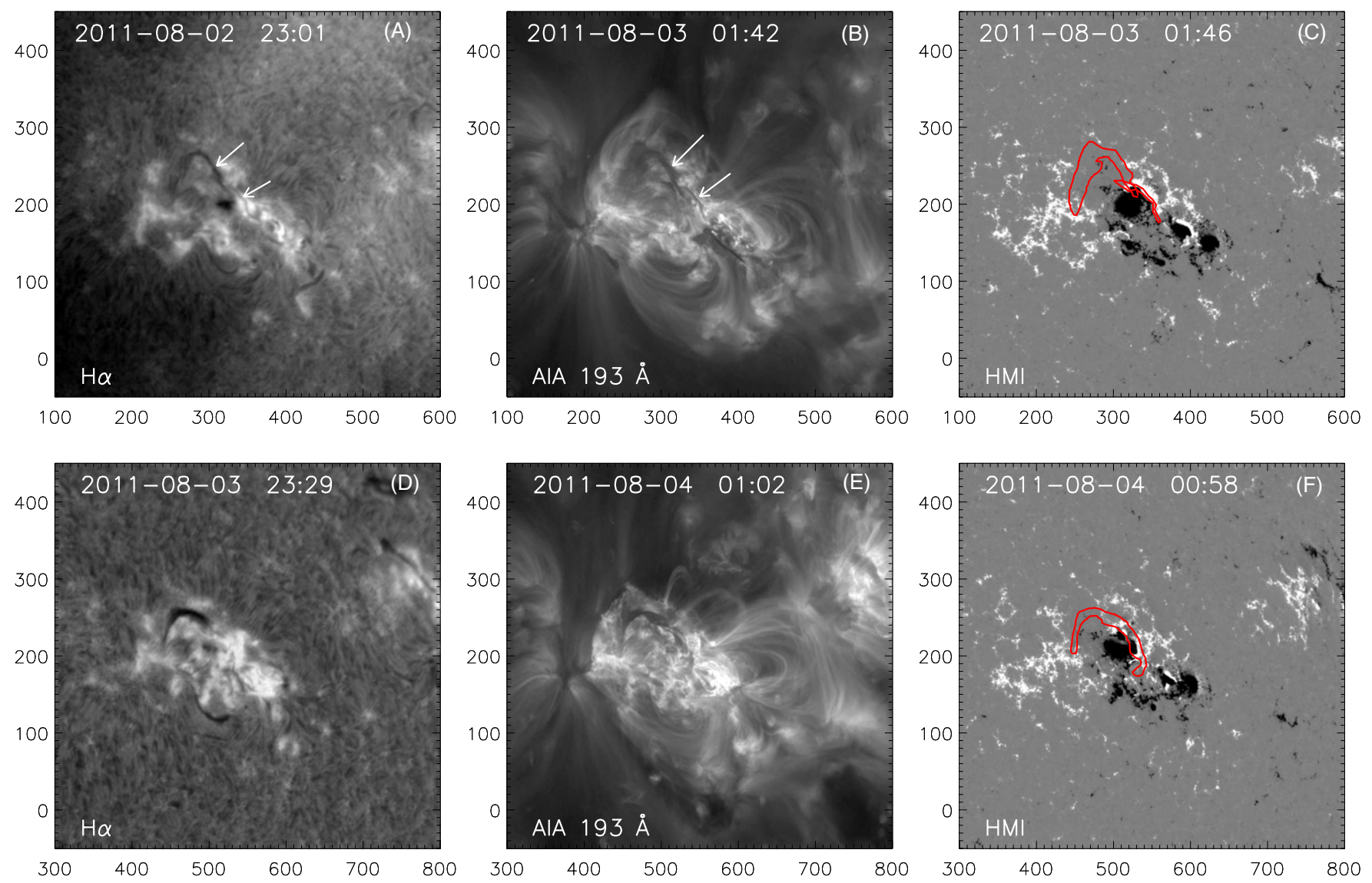

Figure 2. Co-aligned images of the active region taken before (top panels) and after (bottom panels) the M6.0 flare that occurred on 2011 August 3 at 13:17 UT. Left panels: $\mathrm{H} \alpha$ images acquired by the Big Bear Solar Observatory. Middle panels: SDO/AIA images acquired at $193 \AA$ A. Right panels: SDO/HMI magnetograms. The white arrows in the top images indicate the location of two filaments. The red contours on the HMI magnetograms outline the shape of the analyzed filaments derived from the AIA $193 \AA$ images. North is at the top of the images and west to the right.

(A color version of this figure is available in the online journal.)

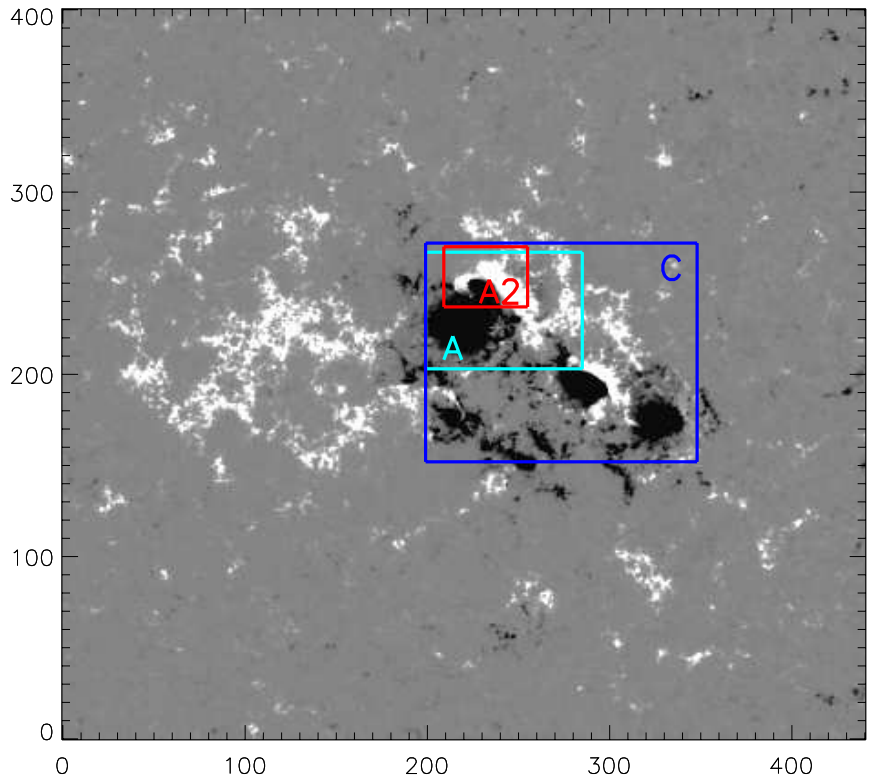

Figure 3. HMI magnetogram of the active region obtained on 2011 August 3 at 00:00 UT. The three squares indicate the three different regions discussed in the text. Region $\mathrm{C}$ is the region that includes the complete sunspot group. Region A is a zoom into the northern sunspot, while Region A2 is a zoom into the locations where the footpoints of the two filaments cross each other. The $x$ and $y$ scales are in image pixels. North is at the top and west to the right.

(A color version of this figure is available in the online journal.) the flux left the region, passing through the lateral boundary. To overcome this difficulty, we used the Yet Another Feature Tracking Algorithm (Welsch \& Longcope 2003) to identify and follow the different magnetic features. In this algorithm, a magnetic structure is identified as a feature only if the magnetic field exceeds a threshold of $50 \mathrm{G}$ and extends to at least 16 pixels for Region A or 8 pixels for Region A2. Among all of the identified features, we consider only those that are tangent to the polarity inversion line at step zero-i.e., on August 3 at 00:00 - and that do not leave the subfield until August 4 at 03:24. However, if one of the tracked features disappears because of either fragmentation or merging and a new feature appears in its place, this latter is also included in our analysis. We then computed the total, positive, and negative flux by summing the magnetic field of all of the features that meet the aforementioned requirements at each time step.

Figure 4 shows the features found using the Yet Another Feature Tracking Algorithm and the respective magnetogram for Regions A (left panels) and A2 (right panels). A significant amount of magnetic flux is canceled at the polarity inversion line and especially along the northern part of the polarity inversion line. This is confirmed by Figure 5, which shows the total (solid blue), positive (dash-dotted red), and negative (dashed black) magnetic flux as a function of time for Region A (top), Region A2 (middle), and Region C (bottom). The vertical line indicates the time of the M6.0 flare; the M9.3 flare occurs just at the end of the observation. 

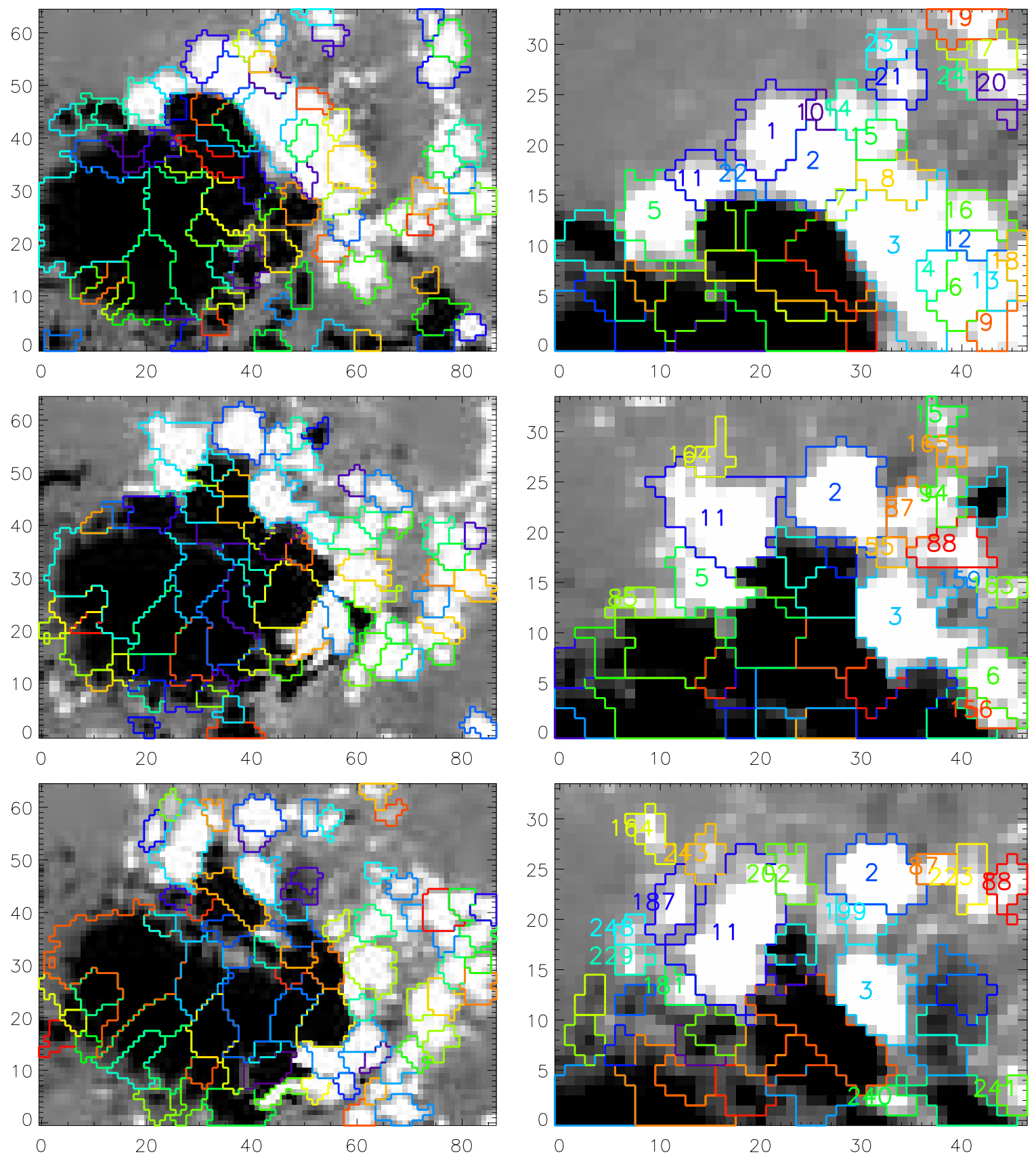

Figure 4. Zoom of the HMI magnetograms in Region A (left panels) and Region A2 (right panels) at different times: 2011 August 3 at 00:00 UT (top panels), August 3 at 18:00 (middle panels), and August 4 at 03:24 (bottom panels). The color contours highlight the features identified using the Yet Another Feature Tracking Algorithm. For clarity, we show only a sample of the magnetic feature labels: those corresponding to the positive flux in Region A2. The magnetic scale is saturated at $\pm 300 \mathrm{G}$. The $x$ and $y$ scales are in image pixels. North is at the top and west to the right.

(A color version of this figure is available in the online journal.)

In Region A, the negative flux increases until August 3 at 05:00 and then decreases slowly until 20:00 on the same day when the decrease becomes much steeper. This trend continues until the onset of the eruption. The flux evolution follows a similar trend in Region A2, where the steep decrease in the positive and negative flux begins only a couple of hours after the M6.0 flare. In contrast, the flux in Region C behaves completely differently. The positive and negative magnetic fluxes remain almost constant throughout the period of observation, although the negative flux increases very slightly.

There is a significant magnetic flux imbalance evident in Figure 5: the negative flux amounts to roughly twice the positive flux. Note that this flux imbalance disappears, however, if we take the dispersed magnetic field in the eastern facula into consideration.

\subsection{Velocity Field}

To derive the photospheric velocity flows, we processed the sequence of co-aligned HMI magnetograms taken between August 3 at 00:00 and August 4 at 03:48 with the Differential Affine Velocity Estimator (DAVE; Schuck 2005) algorithm. This algorithm is a modified local correlation tracking algorithm that also accounts for the contraction, dilation, or rotation of the 

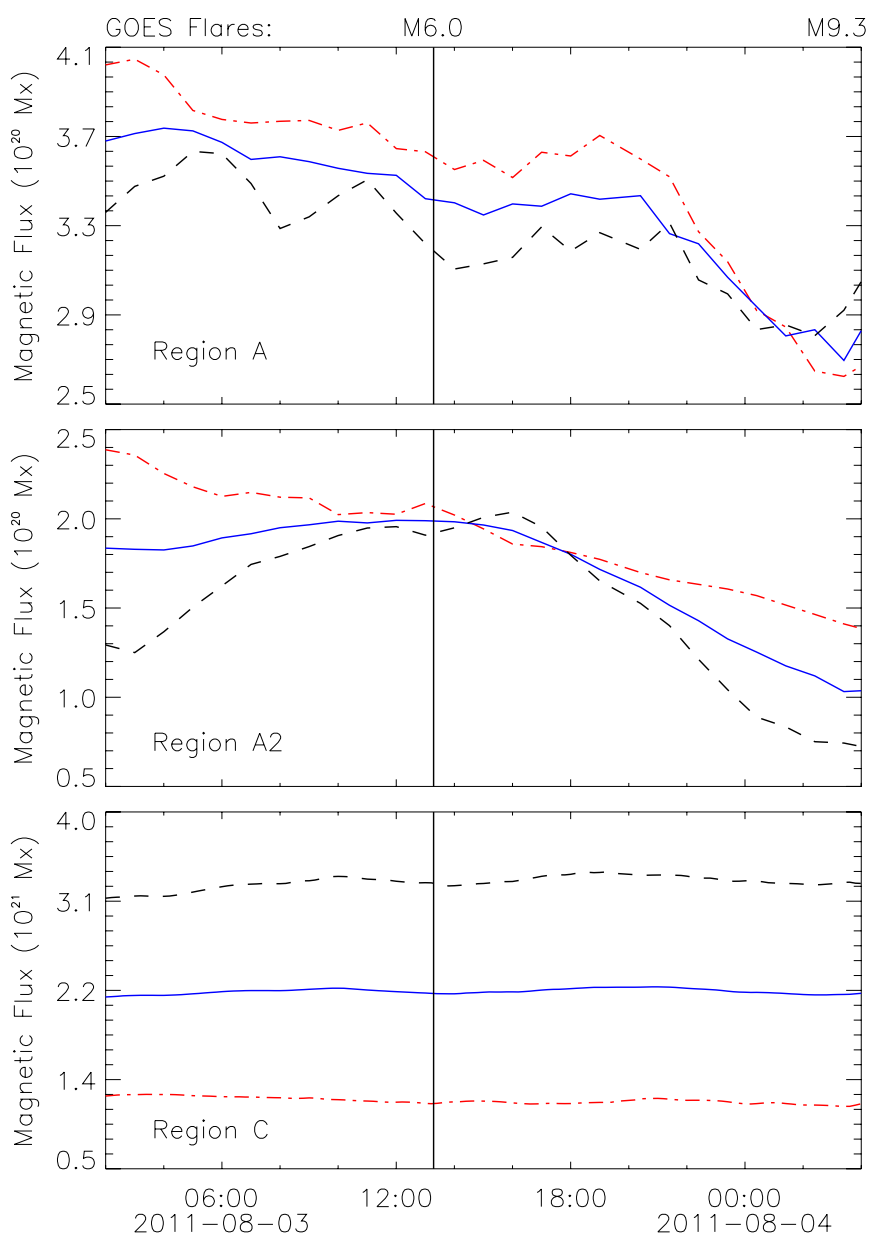

Figure 5. Time evolution of the positive (dash-dotted red), negative (dashed black), and total unsigned (solid blue) magnetic flux for Region A (top), Region A2 (middle), and Region C (bottom).

(A color version of this figure is available in the online journal.)

moving magnetic features and computes a velocity field that is consistent with the vertical component of the induction equation. For this analysis, we used a full width at half maximum of the resolving window of $5^{\prime \prime}$.

Figure 6 shows the velocity field we derived at a few selected instants in time. We observed persistent photospheric shearing motions around the negative polarity of the northern sunspot. These shearing motions are globally directed toward the polarity inversion line of the northern sunspot. However, the northern part of the negative flux distribution is actually subjected to shearing motions directed to the northwest, while the central part of negative flux distribution is subjected to east-west-aligned shearing motions. This trend changes a few hours before the filament eruption; the shearing motions are then mostly directed to the southwest (see Figure 6, bottom panel).

These measured flows strongly support the hypothesis that the observed flux cancellation is a consequence of the convergence shearing motions toward the polarity inversion line of the active region.

Figure 6 also shows that the central negative flux distribution is subjected to persistent, southwest-directed shearing motions, while this is not the case for the southernmost negative polarity, which does not display any significant change in its position. As a consequence, by August 3 at 19:12, the two polarities merge and part of the preexisting (positive) magnetic field is
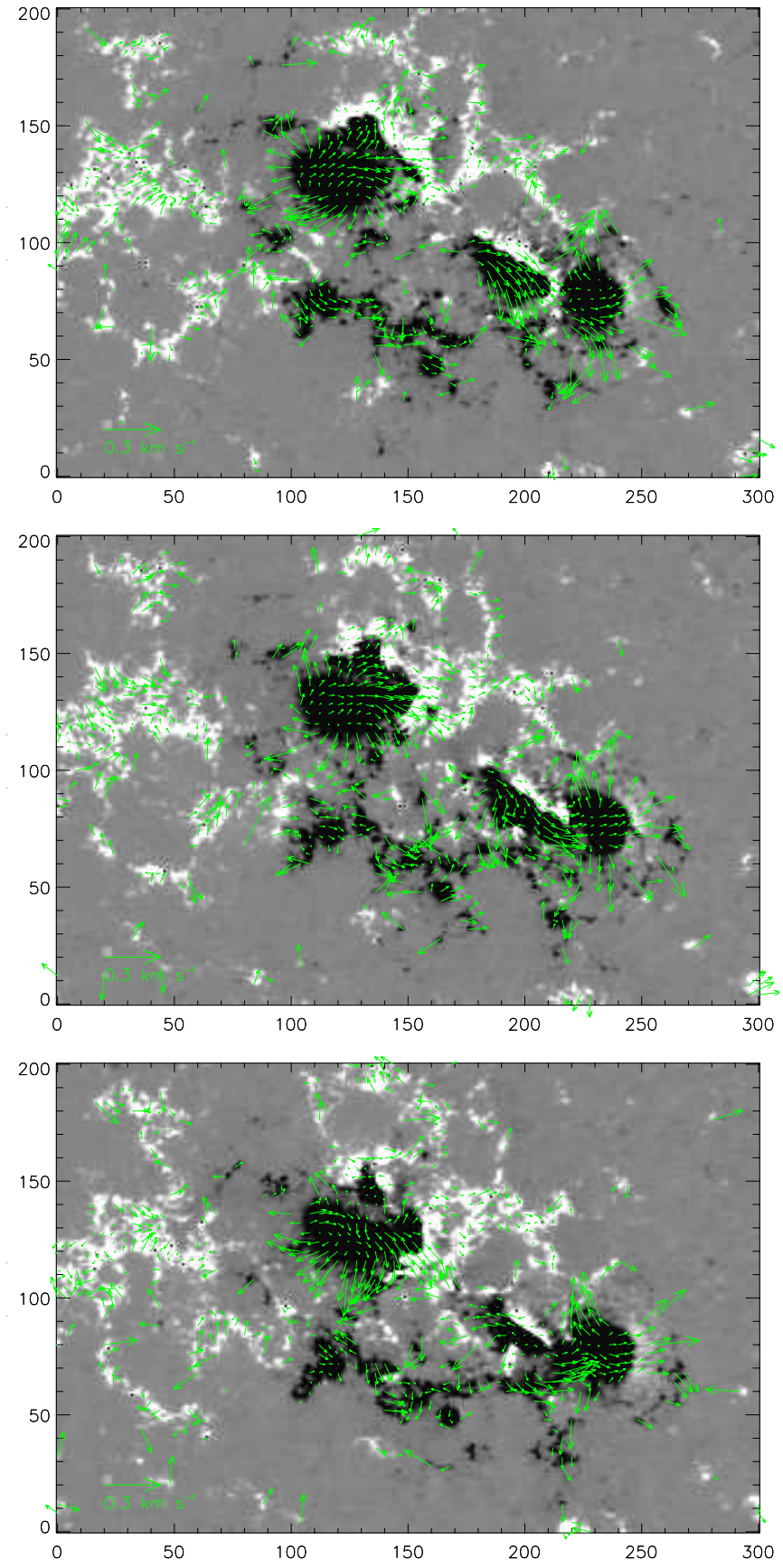

Figure 6. Velocity field derived using the DAVE algorithm for 2011 August 3 11:12 UT (top), 2011 August 3 19:12 UT (middle), and 2011 August 4 02:36 UT (bottom). The $x$ and $y$ scales are in image pixels. North is at the top and west to the right.

(A color version of this figure is available in the online journal.)

canceled. These persistent shearing motions and the associated flux cancellation may have been the responsible for the intense flaring activity in the southern part of the active region (see Section 2).

\subsection{Three-dimensional Reconstruction and Decay Index}

To verify the stability properties of the filament with respect to the torus instability, we used the following procedure. First, we reconstructed the three-dimensional position of the filament at several different times. Second, we computed potential magnetic field extrapolations and calculated the decay index of the 

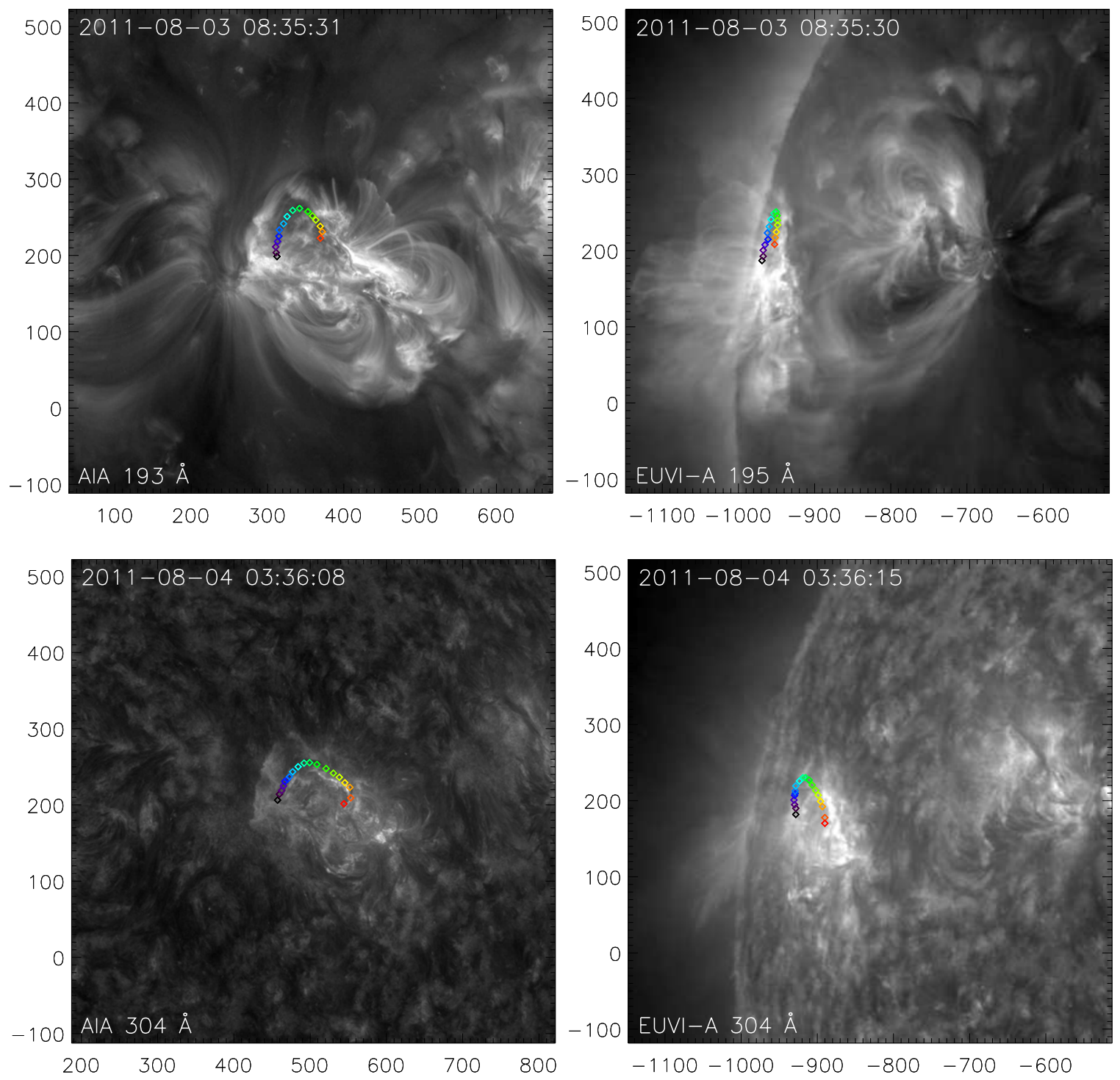

Figure 7. Three-dimensional reconstruction of the filament (color diamonds) projected on AIA $193 \AA$ and EUVI-A $195 \AA$ images obtained on 2011 August 3 at 08:35 UT (top panels), and on AIA $304 \AA$ and EUVI-A $304 \AA$ images taken on 2011 August 4 at 03:36 UT (bottom panels). Diamonds of the same color identify the corresponding structures in each image pair. The $x$ and $y$ scales are arcseconds.

(A color version of this figure is available in the online journal.)

magnetic field at approximately the same times. Last, we compared the height of the reconstructed filament with the height at which the decay index of the field exceeds the critical value $n_{\text {crit }}$. Démoulin \& Aulanier (2010) have shown that for current paths that are not circular and not fully neutralized, the critical value of the decay index $n_{\text {crit }}$ is typically in the $1.2-1.5$ range.

We reconstructed the three-dimensional position of the filament using the triangulation routine scc_measure.pro provided with SolarSoft. This routine, after reading in a pair of images, allows the user to map the line of sight of a point selected in one image of the pair into the field of view of the second image (the so-called epipolar line; Inhester 2006). After the user identifies the intersection between the projected line of sight and the feature of interest, the program can triangulate the feature's three-dimensional location. Because the filament was behind the limb when viewed from STEREO-B, we applied this routine to images from $S D O$ and $S T E R E O-A$.

We performed three reconstructions-first, on August 3 at 08:35, before the M6.0 flare; second, on August 3 at 21:36, about $6 \mathrm{hr}$ after the M6.0 flare; and, third, on August 4 at
03:36, just before the onset of the filament eruption. For the first reconstruction, we used AIA $193 \AA$ and EUVI $195 \AA$ images because the filament was not clearly visible in EUVI $304 \AA$ images because of the bright facula in front of it. However, for the other two reconstructions, we used AIA $304 \AA$ and EUVI $304 \AA$ images because the contours of the filament were best visible in this wavelength. For each of the image pairs, we identified 15-20 points along the filament for the corresponding reconstruction.

Figure 7 shows the reconstructed filament (cross-referenced colored diamonds) projected back onto the corresponding AIA (left panels) and EUVI (right panels) images. The top panels show the reconstructed position of the filament on August 3 at 08:35, while the bottom panels show the reconstructed position of the filament on August 4 at 03:36. The reconstruction shows that the filament is significantly inclined-i.e., the plane containing the axis of the filament is almost parallel to the solar surface. The filament had a maximum height of about $8-10 \mathrm{Mm}$ before the M6.0 flare and of about $14-18 \mathrm{Mm}$ at the moment of the eruption. 


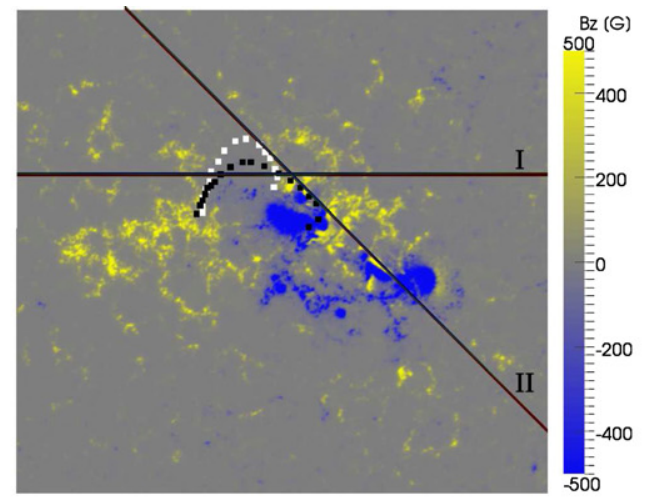

(a)

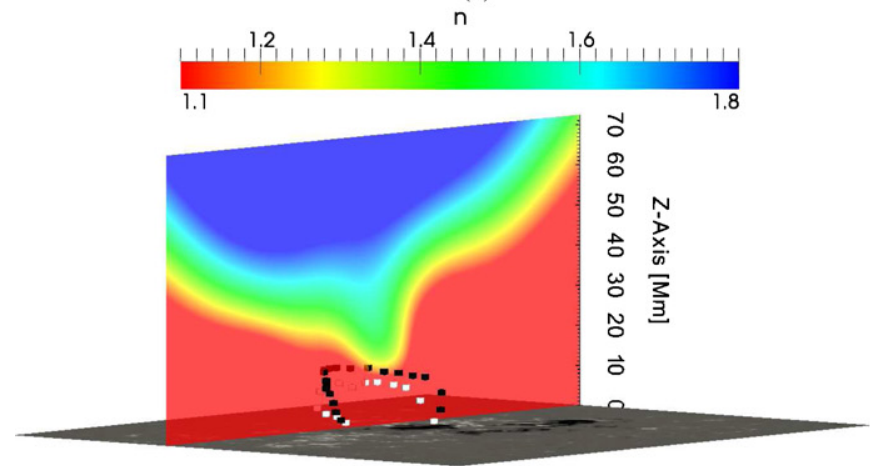

(c)

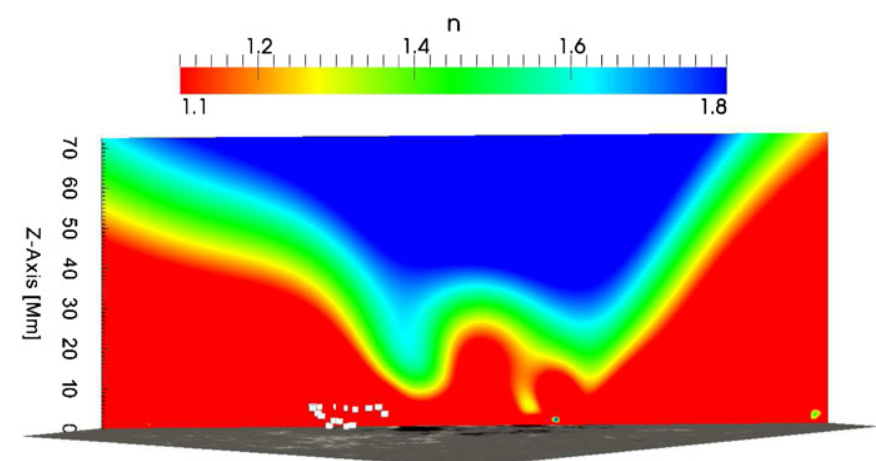

(b)

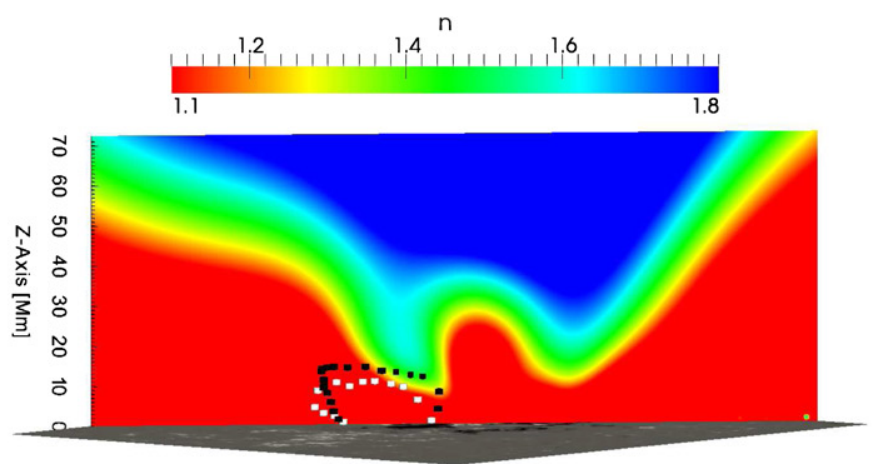

(d)

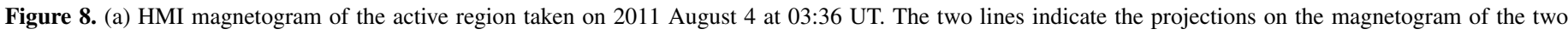

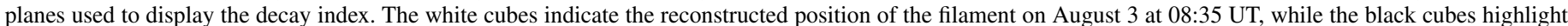

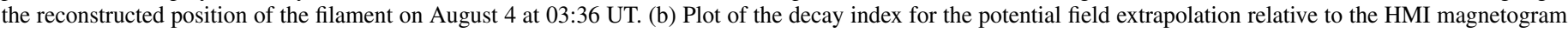

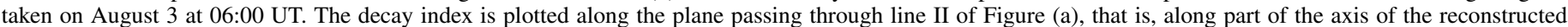

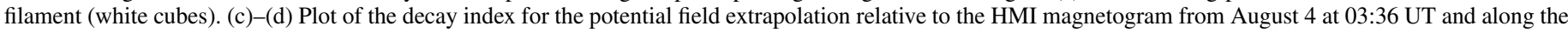

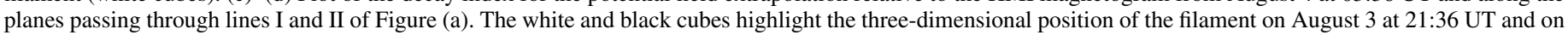
August 4 at 03:36 UT, respectively.

(A color version of this figure is available in the online journal.)

The decay index for a current ring of major radius $R$ and embedded in an external field $B_{\text {ex }}$, is defined as follows:

$$
n=-R \frac{d}{d R}\left(\ln B_{\mathrm{ex}}\right)
$$

where $R$ is the flux rope major radius and $B_{\mathrm{ex}}$ is the magnetic field external to the flux rope (see the Introduction).

With some approximations, Equation (1) can be used to calculate the decay index on the basis of solar observations. First, we estimate the major radius of the flux rope using its peak height above the photosphere (Török \& Kliem 2007; Fan \& Gibson 2007; Aulanier et al. 2010). Second, we use the potential magnetic field as a proxy for the external magnetic field (Guo et al. 2010). To derive the potential magnetic field for the given HMI magnetogram, we use the extrapolation method of Alissandrakis (1981). This method, given the normal component of the magnetic field at the photosphere-i.e., at $z=0$ - uses the fast Fourier transform to calculate the solution of the potential field equation, $\nabla \times \mathbf{B}=0$, in the semi-infinite space $z>0$.

After computing the decay index for the extrapolated potential magnetic field, we insert the reconstructed filament into the extrapolation domain. This approach allows us to compare the three-dimensional position of the filament to the profile of the decay index.

Figure 8 shows the computed decay index along two selected planes for the magnetic field extrapolations performed using the magnetograms obtained on August 3 at 06:00 and on August 4 at $03: 48$. The yellow-green region corresponds to the location where the decay index is $n \simeq 1.3-1.5$ : the height where the system becomes nominally torus unstable.

Figure 8(B) shows the decay index along a plane passing through the compact polarity inversion line of the active region (line II of Figure 8(A)) for the extrapolation on August 3 at 06:00. It is clear from the figure that the region close to the compact polarity inversion line is the most unstable one: in this region, the decay index reaches the critical value at a smaller height. This behavior is also confirmed by Figures 8(C) and (D), which show the decay index along the planes passing through lines I and II of Figure 8(A) for the extrapolation on August 4 at $03: 48$. The behavior of the decay index is a consequence of the topology of the active region. At least close to the filament's location, the magnetic field of the active region can be approximated by two dipoles: first, a large dipole composed of the dispersed positive magnetic field of the facula and the negative polarity of the northern sunspot; and second, a compact dipole composed of the negative polarity of the northern sunspot and the positive polarity to the west of it. The field generated by the compact dipole drops faster with height than the field generated by the large-scale dipole. As a result, the decay index in the proximity of the compact dipole increases more rapidly.

Figure 8 also shows that the decay index does not change significantly between the times of first and the second extrapolation. However, after the M6.0 flare, the morphology of the filament changes and the filament extends into the region where 
the field is more unstable. This change in morphology, and the increased instability of the filament associated with it, is clearly visible when we compare Figure 8(B) with Figures 8(C) and (D).

During the M6.0 flare, the filament (white cubes in Figure 8(B)) is stable with respect to the torus instability; the reconstructed filament is well below the height where the decay index reaches the critical value $n_{\text {crit }} \simeq 1.3-1.5$. However, after the M6.0 flare-more precisely on August 3 at 21:36 - the filament (white cubes in Figures 8(C)-(D)) approaches the region where the decay index of the field is close to the critical value $n_{\text {crit }}$. The filament remains in this location until August 4 at 03:36 UT when it erupts.

The three-dimensional reconstruction of the filament on August 4 at 03:36 (black cubes in Figures 8(C)-(D)) shows that at the moment of the eruption, the filament is in a region where the decay index is in the range of the critical value for the onset of the torus instability.

\section{DISCUSSION}

The filament eruption that occurred on 2011 August 4 is a compelling example of the complexity of coronal dynamics, in which several different phenomena can all contribute to the initiation of a single coronal mass ejection. In this context, it is worthwhile to disentangle which phenomena are essential to causing the eruption-i.e., what, exactly, triggers the eruption-and which phenomena help facilitate the eruption's onset by bringing the system to a point where the trigger mechanism can work. The numerical magnetohydrodynamic simulation performed by Aulanier et al. (2010) shows that the tether-cutting reconnection driven by flux dispersion can facilitate an eruption by bringing the flux rope to a height where the decay index of the overlying field is larger than the critical value for the onset of the torus instability. However, in their simulation, the torus instability is the final trigger mechanism for the eruption.

In the previous sections, we discussed the morphology of the observed filament and its relation to the photospheric magnetic field. As we show in Figure 2, before the M6.0 flare, two filaments (white arrows) are visible both in $\mathrm{H} \alpha$ and AIA $193 \AA$ images. We believe that the two filaments are actually part of the same flux rope that extends from the positive magnetic field of the facula to the southern part of the sunspot. This flux rope also has a significant amount of its flux anchored in the northern part of the sunspot. Complex flux ropes with several footpoints have been discussed by Démoulin et al. (1996) and modeled in numerical magnetohydrodynamic simulations (Zuccarello et al. 2012a). In this case, the additional footpoint of the flux rope may simultaneously support the plasma that constitutes the filaments and introduce significant line-tying effects.

When part of the smaller filament erupted on August 3 at 13:17, resulting in a M6.0 flare, the larger filament was nonetheless unperturbed. To better understand why this filament was relatively unaffected by a significant flare just south of it, we reconstructed the three-dimensional structure of the larger filament a few hours before the flare and compared its position with the decay index for the potential magnetic field. Our results show that the bigger filament was, at that time, in a region that is stable to the torus instability. This may explain why the larger filament did not erupt despite the occurrence of the M6.0 flare close to its western footpoint.

In contrast, the reconnection associated with the flare changed the morphology of the filament (see Figure 2(D)). This reconnection appears to have reduced the line-tying field associated

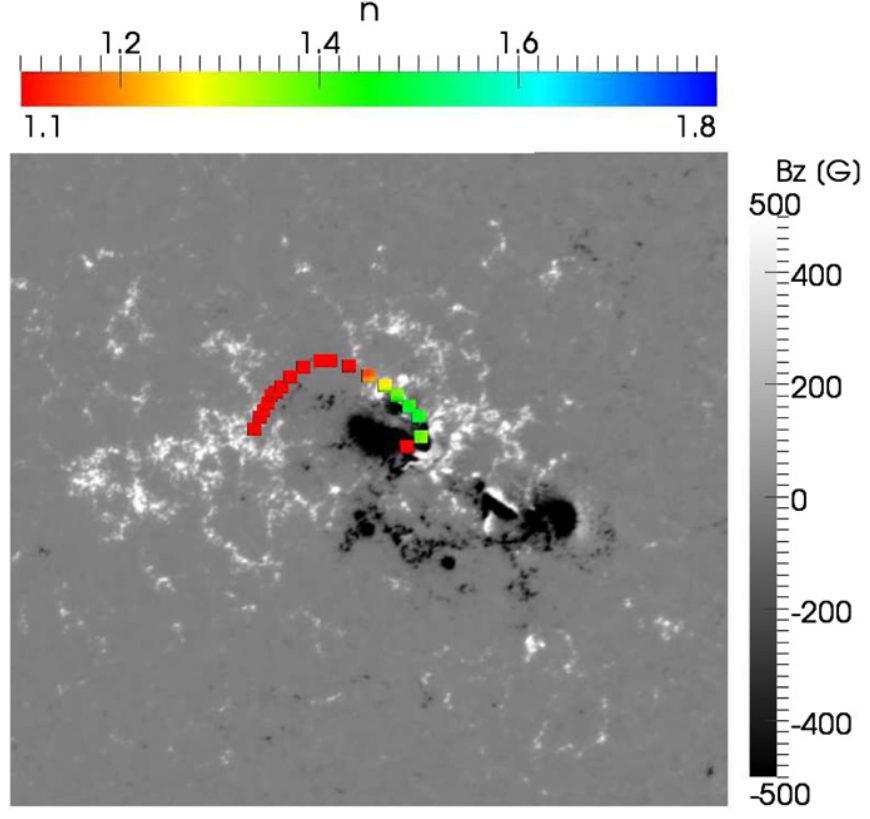

Figure 9. HMI magnetograms of the active region taken on 2011 August 4 at 03:36 UT together with the reconstructed position of the filament on August 4 at 03:36 UT. The reconstructed filament is color-coded with the decay index computed from the potential field extrapolation. North is at the top; west is to the right.

(A color version of this figure is available in the online journal.)

with the flux rope and allowed the plasma to fill the entire flux rope. After the restructuring, the filament grew in length. While it still spanned its original location between the eastern facula and edge of the northern sunspot, it now extended along nearly the full length of the polarity inversion line of the compact dipole composed of the positive-negative polarity of the northern sunspot. This was a fundamental step in the filament's evolution toward eruption. In the region into which the restructured filament then extended, the overlying field scaled much more rapidly with height and, as a consequence, the decay index approached its critical value at lower heights. Figures 8(C)-(D) show that after the M6.0 flare, on August 3 at 21:36, the filament (white cubes) was very close to the torus instability region. However, no eruption occurred until August 4 at 03.48; the filament remained stable for at least $6 \mathrm{hr}$.

The three-dimensional reconstruction of the filament as it was just before the eruption, on August 4 at 03:36, suggests that during the hours that preceded the eruption, the filament underwent a further rise, definitely reaching a height at which the decay index was in the region of the critical value for the onset of the torus instability. During the 8-10 hr preceding the eruption, we observed flux cancellation driven by convergence motions toward the compact polarity inversion line of the northern sunspot. Similar to the dynamics in the magnetohydrodynamic simulations of Zuccarello et al. (2012a), these convergence motions and the associated flux cancellation may have induced tether-cutting reconnection below the filament, which subsequently drove its slow rise. This slow rise abruptly changed at 03:48 when the filament suddenly erupted as a result of torus instability.

Figure 9 shows a top view of the three-dimensional reconstruction of the filament, color-coded using the decay index value at each reconstructed point, and projected onto the HMI magnetogram obtained on August 4 at 03:36. At that time, the filament was located directly above the complex polarity inversion line that extended from the positive magnetic field of 
the facula up to the southern part of the negative polarity of the northern sunspot. It is clear in the figure that the part of the filament that had become torus-unstable at that time (green in the color scale of the figure) is the same part that was directly above the compact polarity inversion line of the northern sunspot. Furthermore, from Figure $1(\mathrm{~F})$, it is clear that this is also the location from which the eruption first began. We believe this is compelling evidence that the torus instability was the trigger of the 2011 August 4 filament eruption.

This research was funded by projects 3E120343 (PDMK/12/ 138, KU Leuven), 1272714N (FWO Vlaanderen), GOA/2009009 (KU Leuven), G.0729.11 (FWO Vlaanderen), and C 90347 (ESA Prodex 9) and the Interuniversity Attraction Poles Programme initiated by the Belgian Science Policy Office (IAP P7/08 CHARM). Additional support for D.B.S. and L.A.R. was provided by the Belgian Federal Science Policy Office through the ESA-PRODEX program, grant No. 4000103240 and by grant FP7/2007-2013 from the European Commissions Seventh Framework Program under the agreement eHeroes (Project No. 284461). The research leading to these results has also received funding from the European Commission's Seventh Framework Programme (FP7/2007-2013) under the grant agreements SOLSPANET (Project No. 269299, www.solspanet.eu), SPACECAST (Project No. 262468, fp7-spacecast.eu), and SWIFF (Project No. 263340, www.swiff.eu). The authors acknowledge the use of data from STEREO/SECCHI and Big Bear Solar Observatory/New Jersey Institute of Technology. The AIA and HMI data are courtesy of NASA/SDO and the AIA and HMI science teams.

\section{REFERENCES}

Alissandrakis, C. E. 1981, A\&A, 100, 197

Amari, T., Aly, J.-J., Luciani, J.-F., Mikic, Z., \& Linker, J. 2011, ApJ, 742, L27
Amari, T., Luciani, J. F., Aly, J. J., Mikic, Z., \& Linker, J. 2003, ApJ, 585, 1073 Aulanier, G., Török, T., Démoulin, P., \& DeLuca, E. E. 2010, ApJ, 708, 314 Bateman, G. 1978, MHD Instabilities (Cambridge, MA: MIT Press)

Chen, P. F. 2011, LRSP, 8

Démoulin, P., \& Aulanier, G. 2010, ApJ, 718, 1388

Démoulin, P., Priest, E. R., \& Lonie, D. P. 1996, JGR, 101, 7631

Fan, Y., \& Gibson, S. E. 2007, ApJ, 668, 1232

Forbes, T. 2000, in Solar Flare Models, ed. P. Murdin

Forbes, T. 2010, in Models of Coronal Mass Ejections and Flares, ed. C. J. Schrijver \& G. L. Siscoe (Cambridge: Cambridge Univ. Press), 159

Forbes, T. G., \& Isenberg, P. A. 1991, ApJ, 373, 294

Forbes, T. G., Linker, J. A., Chen, J., et al. 2006, SSRv, 123, 251

Forbes, T. G., \& Priest, E. R. 1995, ApJ, 446, 377

Guo, Y., Ding, M. D., Schmieder, B., et al. 2010, ApJ, 725, L38

Howard, R. F., Harvey, J. W., \& Forgach, S. 1990, SoPh, 130, 295

Inhester, B. 2006, arXiv Astrophysics e-prints

Isenberg, P. A., Forbes, T. G., \& Demoulin, P. 1993, ApJ, 417, 368

Kaiser, M. L., Kucera, T. A., Davila, J. M., et al. 2008, SSRv, 136, 5

Kliem, B., \& Török, T. 2006, PhRvL, 96, 255002

Klimchuk, J. A. 2001, in Geophys. Monograph Series, Vol. 125, Space Weather, ed. P. Song, H. J. Singer, \& G. L. Siscoe (Washington, DC: American Geophysical Union), 143

Lemen, J. R., Title, A. M., Akin, D. J., et al. 2012, SoPh, 275, 17

Lin, J., \& Forbes, T. G. 2000, JGR, 105, 2375

Mackay, D. H., \& van Ballegooijen, A. A. 2009, SoPh, 260, 321

Pesnell, W. D., Thompson, B. J., \& Chamberlin, P. C. 2012, SoPh, 275, 3

Roussev, I. I., \& Sokolov, I. V. 2006, in Geophys. Monograph Series, Vol. 165 , Solar Eruptions and Energetic Particles, ed. N. Gopalswamy, R. Mewaldt, \& J. Torsti (Washington, DC: American Geophysical Union), 89

Schou, J., Scherrer, P. H., Bush, R. I., et al. 2012, SoPh, 275, 229

Schuck, P. W. 2005, ApJ, 632, L53

Seaton, D. B., Mierla, M., Berghmans, D., Zhukov, A. N., \& Dolla, L. 2011, ApJ, 727, L10

Stern, R. A., Shing, L., Catura, P. R., et al. 2004, Proc. SPIE, 5171, 77

Titov, V. S., \& Démoulin, P. 1999, A\&A, 351, 707

Török, T., \& Kliem, B. 2007, AN, 328, 743

van Tend, W., \& Kuperus, M. 1978, SoPh, 59, 115

Welsch, B. T., \& Longcope, D. W. 2003, ApJ, 588, 620

Wuelser, J.-P., Lemen, J. R., Tarbell, T. D., et al. 2004, Proc. SPIE, 5171, 111

Zuccarello, F. P., Meliani, Z., \& Poedts, S. 2012a, ApJ, 758, 117

Zuccarello, F. P., Romano, P., Zuccarello, F., \& Poedts, S. 2012b, A\&A, 537, A28 


\section{Queries}

\section{Page 11}

Q1

Author: Please check the details for any journal references that do not have a pale purple link (CrossRef doi) or a blue link (NASA ADS or arXiv e-print) in the two-column proof (articlestyle layout). A journal reference with no links may contain some incorrect information.

Q2

Author: Please confirm whether in reference "Bateman (1978)" the publisher details are correct as included.

Q3

Author: Please provide the volume or page number, as applicable, in reference "Chen (2011)."

Q4

Author: Please provide publisher details (name and location) and page number in reference "Forbes (2000)."

Q5

Author: Please provide the arXiv number for reference "Inhester (2006)," and kindly also update the same if possible.

Q6

Author: Please confirm whether in references "Klimchuk (2001)" and "Roussev \& Sokolov (2006)" the publisher's location is correct as included.

\section{Online-only colour figures}

This proof PDF is identical in specification to the PDF file that will be published in the online journal. To view any online-only color figures as they will appear in the printed journal, we recommend that this color PDF file be printed on a black \& white printer. 